\title{
THERMOBASE - A New Medical Device to Aid the Control and Temperature of Liquid Meals for Post Bariatric Surgery Patients
}

\author{
Rafael Carneiro G. Fernandes \\ Graduate Program in Applied \\ Informatics PPGIA/UNIFOR \\ Avenue Washington Soares, 1321, \\ Fortaleza-CE, Brazil
}

\author{
Juliana Martins de Oliveira \\ Graduate Program in Applied \\ Informatics PPGIA/UNIFOR \\ Avenue Washington Soares, 1321, \\ Fortaleza-CE, Brazil
}

\author{
Victor Hugo C. de \\ Albuquerque \\ Graduate Program in Applied \\ Informatics PPGIA/UNIFOR \\ Avenue Washington Soares, 1321, \\ Fortaleza-CE, Brazil
}

\begin{abstract}
Bariatric surgery is a surgical procedure in order to promote the isolation of the stomach and thereby reduce the health risks arising from obesity. The meal in the first month after surgery, which may extend for a longer period according to experts, is based only on a liquid diet. During this period of rehabilitation, the patient ingests $20 \mathrm{ml}$ of fluid every 10 minutes, and should be followed strictly for proper hydration throughout the day. Liquids like soups, juices and waters, are part of diet and needing to be stored in a container such as a bottle, which generally can do not store them in the correct temperature to facilitate the intake for nutritional education caused by diet on postoperative of bariatric surgery throughout the day. This document aims to present a project to improve the quality of life of people undergoing surgery to reduce the stomach, through the development of a device called adjustable THERMOBASE, wherein the patient is able to control the temperature of the heating fluid or cooling the machine contact surface, which should be in contact with a container containing the liquid to be consumed according to the model proposed by nutritionists that to monitor post operative diet. Thus, by means of physical property of thermal conduction, through the Peltier sensor, the soup can be heated to the boiling point or fresh juice to its freezing point, as examples of the extreme draft potential. The THERMOBASE tells you how many drinks were consumed during the day providing valuable nutritional information for nutrition professionals who follow the patient postoperatively, and audible, visual or vibration alarm, indicating the next meal time deadline.
\end{abstract}

\section{General Terms}

Period of rehabilitation bariatric postoperative, device for nutrition reeducation.

\section{Keywords}

Peltier sensor, bariatric surgery, obesity, quality of life.

\section{INTRODUCTION}

Second Ginter and Simko, in [1], about 1/3 of the adult population worldwide suffers from obesity. $\mathrm{Ng}$ et al., [2], from Global Burden of Disease conducted by the Institute for Health Metrics and Evaluation at the University of Washington, conducted a research in 188 countries through censuses and statistical research papers in all areas of the planet between 1980 and 2013, analyzing studies in children, adolescents and adults in the world, there are 2.1 billion overweight or obese people, representing almost $30 \%$ of the population. In 1980, that number was 857 million. The increase in obesity in the last three decades occurred in all regions of the world, representing a public health problem in rich and poor countries.

The obesity is considered a chronic disease that can be caused by several factors, e.g., anxiety, depression, bad food, genetic predisposition, customs, culture and lack of physical activities. The decision to undergo the surgical procedure is responsibility the patient and a large team of skilled health professionals from different areas of expertise, such as such as pulmonology, cardiology, nutrition, endocrinology, psychology, physical therapy, among others. Several comprehensive medical examinations and tests must be performed to judge the real need and possible risks of the procedure, [3].

The period of the first month after surgery is critical and considered critical to the success of surgery The patient can not eat solid food for being sutured organs that come in direct contact with food. This situation can be considered critical because of the risks of contracting infections and may even lead to death. Second Tayne et al., in [4], show through a survey of patients who have undergone bariatric surgery, show that the majority of readmissions occurred within the first week after discharge. Most readmissions that occur in the first week post-discharge are most commonly associated with dehydration, pain or wound. For the incidence of readmission reduction, nutritional education of the patient and the monitoring of the health team both physical and mental are fundamental in pre and postoperative periods. In this period the food of the individual must be strictly the Liquids base, once the stomach and intestines are still sutured, and consequently, with restriction of food that may be eaten.

In some cases, the patient must take $20 \mathrm{ml}$ of liquid nutrient and hydrating the interval of 10 minutes, being established by staff which takes into account an adequate hydration throughout the day. The ingestion of the meal, in certain range, must be between three to five small sips, In addition to condition the patient to eat meals in a slow pace so that the taste of foods is felt, and the sensation of satiety with a smaller amount of food than usual, [5]. Due to this restriction, bariatric surgery has been associated with nutritional deficiency factors. Second Tack e Deloose, in [6], a common factor to all bariatric surgery is the reduction of the total nutrient intake (early) in the postoperative phase. This reduced food intake may be associated with reduced consumption of specific micronutrient, how do B Complex vitamins, A vitamin Many patients undergoing reduction of the stomach do not have a care in the postoperative period to assist with all meals, forcing them to store your food in bottles at your fingertips, promoting an unwanted heat transfer between the liquid food and the environment in which it is 
located. These thermal exchange, are often inadequate to the patient, since the heat can juices and soups cool down beyond that desired by the same, for example. When they are at ambient temperature can make it more difficult ingestion, especially in the first few weeks, wherein the liquid food are exclusively without fibers, buds or processed vegetables.

In this context, this paper developed a prototype, called THERMOBASE, with the goal of improving the quality of life in the postoperative period comprising the first two months of people undergoing bariatric surgery and thus accelerate their rehabilitation collaborating for a nutrition and adequate hydration during this critical period of change and dietary adaptations. With this device, the patient can control the temperature of the liquid to be ingested, or cool a heat juice or a soup for example, through the Peltier sensor, and consists of a configurable buzzer, whose function is to remember the exact food intake the next time and store and indicates the number of servings consumed in the period since. The device has too some extra accessories such as cupholder, Light Emitting Diode (LED) to illuminate it and control the amount and type liquid food ingested throughout the day, allowing to generate graphs based on hydration and nutrition types of food pre-registered in more detail by means of program properly installed on a personal computer.

\section{METODOLOGY}

In this section, we describe the three steps involved in the development of THERMOBASE, either: thermal base, circuit electronics and software. The Figure 1 illustrates the flow chart of the steps considered for development of the design and use by the patient.

\subsection{Thermal Base}

The thermal base consists of a ceramic wafer consisting of a thermocouple known by the name of the discoverer of the phenomena involved in the Peltier sensor, which consists in producing a temperature gradient across two junctions of two different conductors or semiconductors composition, when they are subjected to an electrical voltage in a closed loop, which was applied a thermal conductive, paste based on silver to optimize the heat exchange between the chip and the heat sink (made of aluminum), that in turn has an attached small cooler that consists of an electromechanical fan designed to dissipate heat in electronics aiding in heat transfer. To sustain the equipment, we use supports comprised of aluminum and plastic, one screwed to the heatsink to receive a bottle with various meals bottle rack mass, as illustrated in Figure 2. The base has a LED to illuminate the equipment in the absence of another source of light and a small cup of aluminum to be used in contact with an optimized heat transfer surface, allowing better conduction between the base and the liquid, Figure 3 .

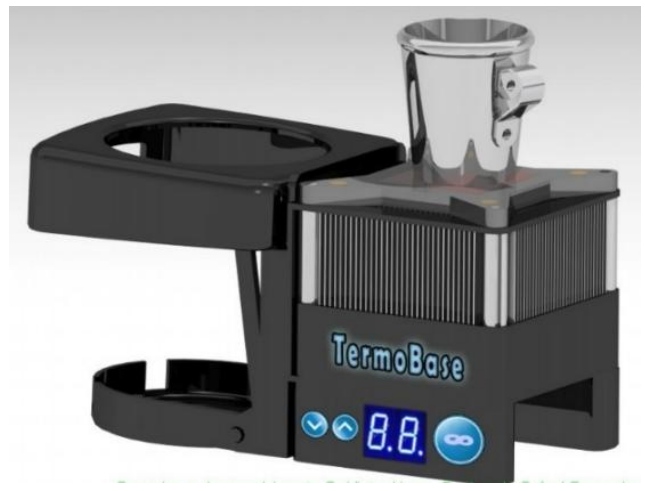

Figure 2: 3D modeling THERMOBASE.

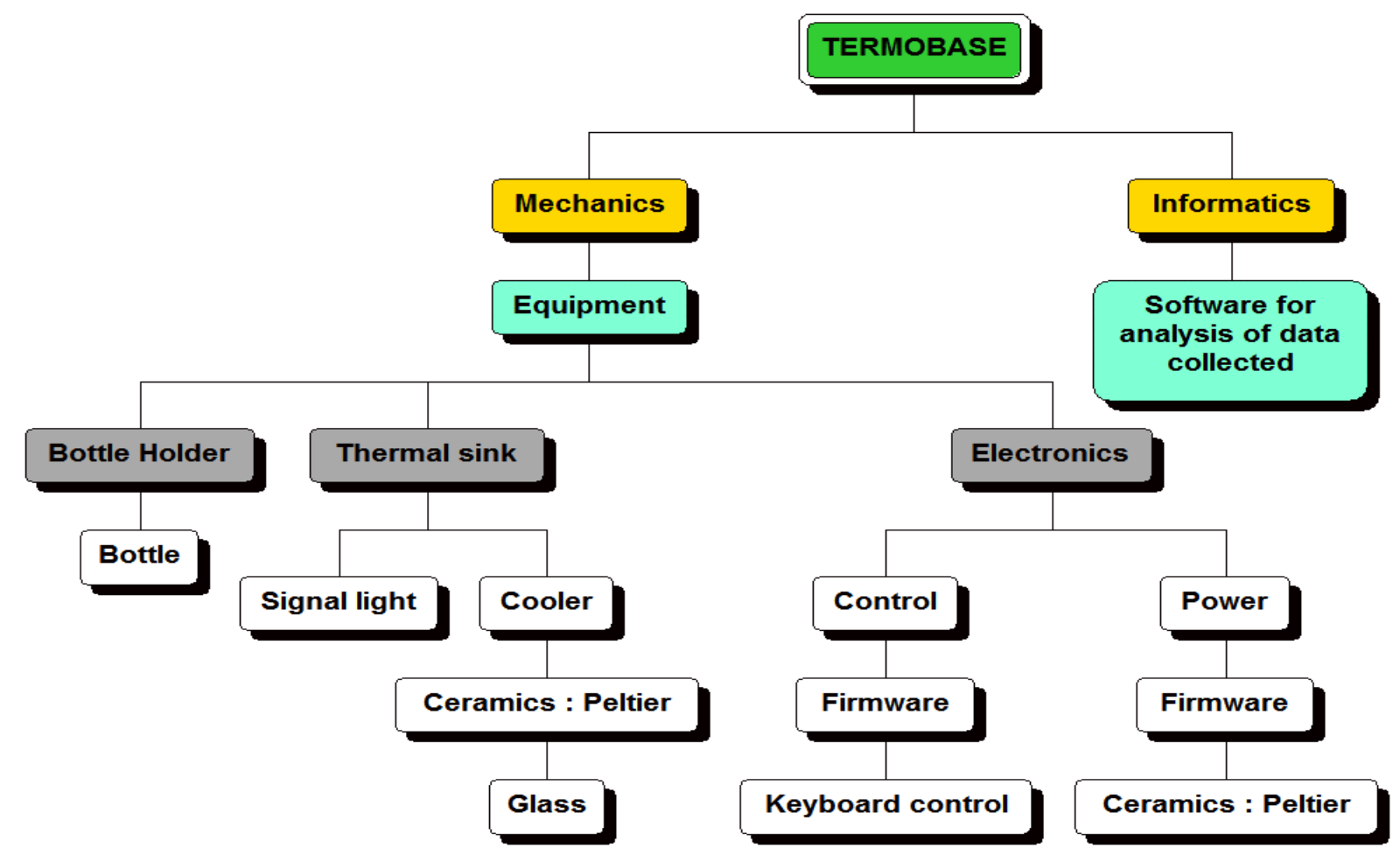

Figure 1: Flowchart of steps for the development of THERMOBASE. 


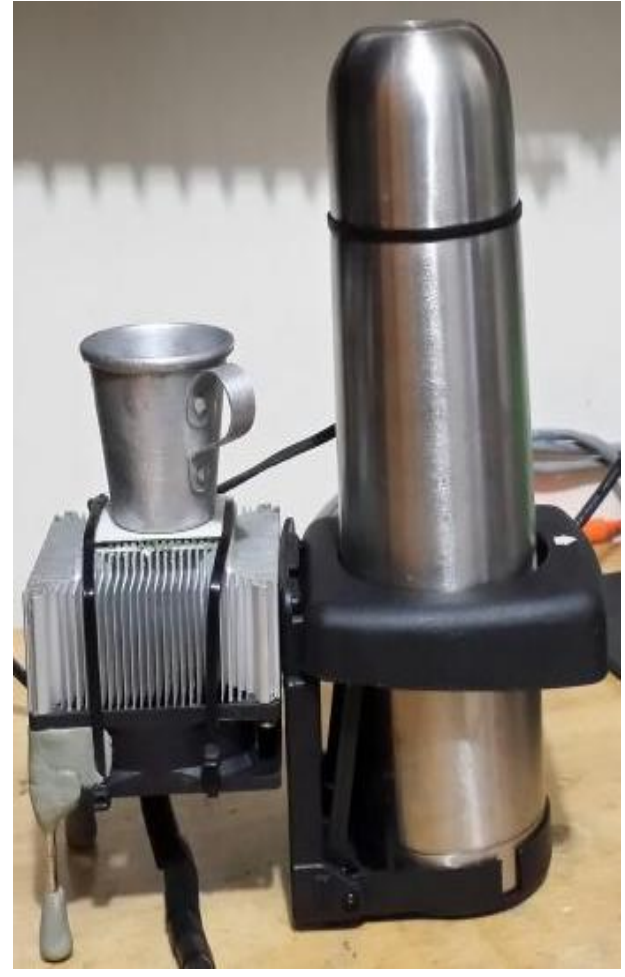

Figure 3: Thermal Base operating THERMOBASE.

The chip used Peltier sensor has a contact area of $16 \mathrm{~cm}^{2}$ and a thermal power $\mathrm{W}$, reaching a temperature variation of $67^{\circ} \mathrm{C}$, consuming a maximum current of $3 \mathrm{~A}$ at a voltage of $15,2 \mathrm{~V}$. The heat exchange between one face of the wafer and the other is directly related to the ability of heat exchange between the heat sink, which increases the contact area of one face with the ambient air in the environment that is, [7]. The cooler optimizes thermal exchange sink, obtaining elevated temperatures up to $140^{\circ} \mathrm{C}$ and relatively low temperatures of around $-5^{\circ} \mathrm{C}$. The cooler used has dimensions of $8.0 \times 8.0 \mathrm{x}$ $2.5 \mathrm{~cm}^{3}$ rotation $2500 \mathrm{rpm}$ and $12 \mathrm{~V}$ voltage. The heatsink made of aluminum, has a volume of approximately $200 \mathrm{~cm}^{3}$, possessor of several fins which increase the surface area of contact with the ambient air to facilitate heat Exchange. Finally, a small reservoir of liquid (I done aluminum) with a capacity of $50 \mathrm{ml}$.

\subsection{Electronic Circuit}

The electronic circuit has, as a major component of work, a microcontroller from Microchip (PIC18F2550A), responsible for controlling the temperature of the base exchanging the relays controlling the voltage of the wafer and measuring the resulting new temperature. An integrated circuit (IC) informs LM35 temperature of the wafer in the alphanumeric display two lines and 16 characters per line. The microcontroller is also responsible for measuring the amount of ingested fluid throughout the day, in addition to producing an electrical signal to the speaker that has the function of alarm (remember) the patient ingesting the next meal. The circuit has Universal Serial Bus (USB) connectivity responsible for communication between a computer and the microcontroller via USB v 1.1 / 2.0 / 3.0, allowing supply a spreadsheet that generates a graph of daily hydration allowing also change the ringing alarm. The circuit also comprises some resistors, capacitors, LED status indicators by visual means, diodes, buttons, and among others, as illustrated in Figure 4.

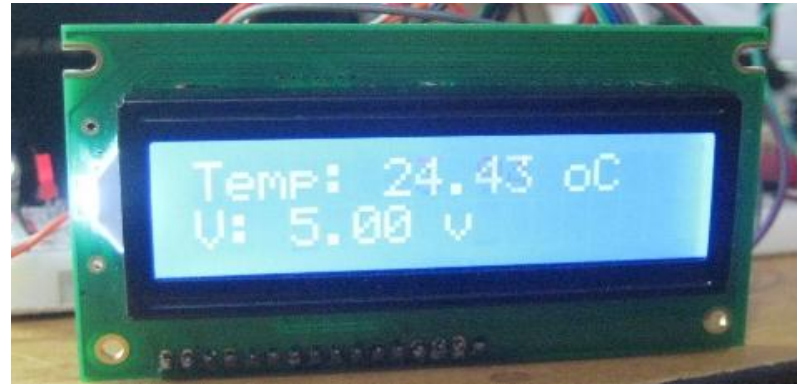

Figure 4: Information in real time on THERMOBASE.

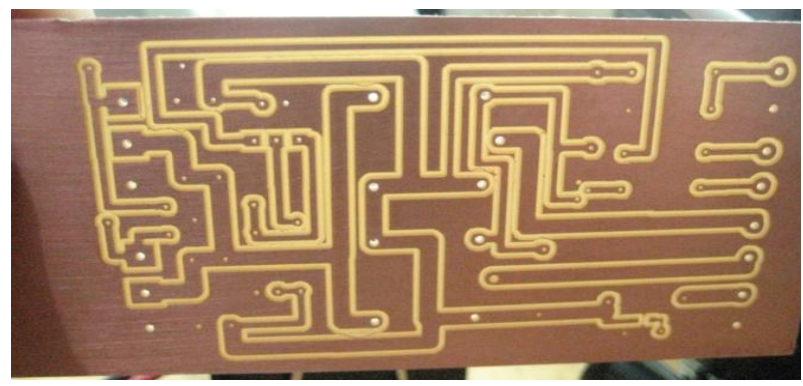

Figure 5: Electronic circuit board THERMOBASE.

The supply voltage of the circuit is $12 \mathrm{~V}$ and can be obtained from an external source to supply at least DC current $5 \mathrm{~A}$ or a sealed battery typically used in residential alarm center, which has a range of approximately two hours of continuous use, still having the option to be connected to the cigarette lighter socket of vehicles that operate on the same voltage required by the project, which is fully converted to $5 \mathrm{~V}$ to power parts of the circuit as the microcontroller, e.g., directed and without alteration for relay switches the poles and PWM modulated by changing the voltage between $1 \mathrm{~V}$ and $12 \mathrm{~V}$ for tablet Peltier, [8].

\subsection{Softwares}

The THERMOBASE has two different softwares. The first is a firmware, responsible for all control and data storage of thermal base, and the second, called Monitor software, which is installed on a personal computer and is responsible for collecting the data base and generate graphs and statistics hydration/nutrition of the patient. The firmware is developed in $C / C^{++}$language using the $\mathrm{C} 18$ compiler is responsible for linking each button its specific function by changing the temperature, regulating the alarm and/or lighting a small light to illuminate the base when needed, it has the function of storing the amount of liquid ingested. Referring to the operating frequency of the crystal used in the microcontroller's firmware is able to work at well-defined intervals of time, allowing high precision buzzer. Natively microcontroller peripheral has USB serial communication, it is possible to obtain two-way communication between the microcontroller and personal computer (PC) so that settings and exchange of statistical data are accomplished. The PC software is very simple and intuitive, and compiled being written in Borland Delphi $7^{\mathrm{TM}}$ with the function of establishing the ASCII standard serial communication between a computer and a thermal basis. Thus, the software receives the statistical data and organizes them into a text file in order to be interpreted by the EXCEL ${ }^{\mathrm{TM}}$ spreadsheets reader, generating graphs and percentages of hydration. The software also has a field to enter the settings for maximum and minimum temperatures range from triggering the alarm syncing with THERMOBASE. 


\section{RESULTS AND DISCUSSION}

This study aimed to develop of a device to help post-bariatric surgery patients in ingesting more satisfying for daily food nutrition of small doses of hot and cold fluids throughout the day and check the quantities ingested determined in the diet. Patients and nutritionists who analyzed the tool through a presentation, indicated positive factors for the use of THERMOBASE during the postoperative period, as well as leave liquids in pleasant temperatures for consumption, marking the amount of liquid ingested daily gives the patient control that is following the diet correctly. Being effective for its size making it portable, versatile and low cost, easy use.

\section{CONCLUSIONS}

The THERMOBASE proved to be very useful, according to the opinion of three patients underwent surgical process to reduce stomach, besides the experts involved in the area. However, the miniaturization of electronic circuitry and an engineered product are most appropriate features that should add value to the project. Once a design is manufactured manually, the few pieces of laundry is not possible, for example, a support and insulation for packing a mug, avoiding loss of thermal energy converted by THERMOBASE.

The Thermal Conversion Efficiency of chip not used prototype and approximate 50\%, a reasonable efficiency, but it can improve with getting gums more modern. Using the System for more than two hours with sealed batteries makes it almost the unfeasible due to weight and volume of the High and Lead acid batteries. With the passage of the liquid phase of food, the owner of the base system can be used to cool cans of various types of beverages or solid foods their heat the wrapping foil to distribute the heat evenly, maintaining THERMOBASE useful in everyday.

In cases of morbid obesity in which surgical intervention is required, several tools and techniques complement and assist the postoperative period, reinforcing the psychological counseling and helping the patient to remain in the plan of conduct postoperatively. Thus, THERMOBASE helps reduce the discomfort of the patient to take liquids in undesired temperatures, taking into account the need to ingestion thereof.

Fluid intake in temperature that pleases the user, stimulates maintain strict diet, as proposed by the THERMOBASE project, it provides juices, water, coconut milk and skim milk always cold to taste, and warm broths and soups that correspond to the main meals. Still serves as a hydration/nutrition and reminds the user to monitor the time of the next drink.

\section{ACKNOWLEDGMENTS}

The second and last authors thank Coordination for the Improvement of Higher Education Personnel (CAPES) and National Council for Research and Development (CNPq) via grant \# 470501/2013-8.

\section{REFERENCES}

[1] E. Ginter and V. Simko. Recent data on obesity research: $\beta$-aminoisobutyric acid. Bratisl Lek Listy. 115 (8), 492493, 2014.

[2] Marie NG et al. Global, regional, and national prevalence of overweight and obesity in children and adults during 1980-2013: a systematic analysis for the Global Burden of Disease Study 2013. The Lancet. 384(9945), 766-781, 2014.

[3] C. Benedetti. Obesity and slimming: a study on morbidly obese submitted to gastroplasty. Master in Clinical Psychology at Pontifícia Universidade Católicade São Paulo, São Paulo, 2001.

[4] S. Tayne, C. A. Merrill, S. N. Shah, J. Kim and W. C. Mackey. Risk Factors for 30-Day Readmissions and Modifying Postoperative Care after Gastric Bypass Surgery. Journal of the American College of Surgeons. 219 (3), 489-495, 2014.

[5] A. B. Garrido Jr. Situações especiais: tratamento da obesidade mórbida. In: Halpern A, Matos AFG, Suplicy HL, Mancini MC, Zanella MT, editores. Obesidade. São Paulo: Lemos Editorial, 331-340, 2002.

[6] J. Tack and E. Deloose. Complications of bariatric surgery: Dumping syndrome, reflux and vitamin deficiencies. Best Practice \& Research Clinical Gastroenterology. 28 (4), 741-749, 2014.

[7] G. S. Nolas, J. Sharp and J. Goldsmid. Thermoelectrics: Basic Principles and New Materials Development, Springer-Verlag, Berlin, 2001.

[8] N. C. Braga. Mechatronics Sourcebook. Delmar Learning, 1 edition, Clifton Park, NY, 2002. 